\title{
Dynamic ultrafast CZT imaging: Time for a paradigm change in myocardial perfusion imaging
}

\author{
Riccardo Liga, $M D$, ${ }^{a}$ and Alessia Gimelli, $M D^{b}$ \\ a Università di Pisa, Pisa, Italy \\ b Fondazione Toscana G. Monasterio, Pisa, Italy
}

Received Jan 21, 2020; accepted Jan 21, 2020

doi: $10.1007 / \mathrm{s} 12350-020-02051-1$

\section{See related article, pp. 2518-2529}

Cardiac stress imaging has been always regarded as the best way for the non-invasive assessment for the presence of significant coronary artery disease (CAD), guiding patients' risk stratification and clinical management. ${ }^{1}$ Accordingly, for over half a century, decisions regarding the opportunity of submitting patients to complex and often risky coronary interventions, including both percutaneous and surgical procedures has been based on the results of an imaging stress test. Reports based on historical data had always attributed to all the different stress cardiac imaging techniques as an excellent accuracy in detecting CAD, with crude values of sensitivity and specificity in the range of $90 \% .^{2}$ In this setting, myocardial perfusion imaging (MPI) with single-photon emission computed tomography (SPECT) has been generally regarded as a reference standard, allowing the evaluation of myocardial ischemia in the majority of patients and clinical settings, and maintaining an adequate diagnostic accuracy even in "extreme" conditions (i.e. morbid obesity, end-stage renal disease/dialysis, respiratory failure). ${ }^{3,4}$

However, data accumulated in the last decade have somehow challenged these concepts, demonstrating that, in present days, the accuracy in detecting CAD of the currently available non-invasive cardiac stress imaging modalities is, at best, moderate, missing the diagnosis of hemodynamically significant coronary lesions in a

\footnotetext{
Reprint requests: Alessia Gimelli, MD, Fondazione Toscana G. Monasterio, Pisa, Italy; gimelli@ftgm.it

J Nucl Cardiol 2021;28:2530-2.

$1071-3581 / \$ 34.00$

Copyright (C) 2020 American Society of Nuclear Cardiology.
}

significant proportion of the cases. ${ }^{5}$ Those findings were further reinforced by the results of the PACIFIC study, reporting the unacceptably low sensitivity $(57 \%)$ of conventional SPECT imaging in detecting significant CAD on invasive coronary angiography (ICA) + fractional flow reserve (FFR), despite a moderate specificity $(84 \%)$ and overall accuracy $(77 \%){ }^{6}$ As a result of those data, the recent NICE recommendations on the management of patients with suspected or known stable CAD have emphasized the assessment of coronary anatomy over myocardial perfusion, pointing to the use of computed tomography coronary angiography (CTCA) rather than MPI, ${ }^{7}$ an indication shared by other clinical guidelines particularly in patients at low-intermediate pre-test likelihood of CAD. The reasons for these somehow disappointing findings are twofold. First of all, MPI is only able to demonstrate the presence of hemodynamically significant coronary lesions, unable to detect non-obstructive or diffuse coronary atherosclerosis, which can have an adverse prognostic impact, and deserve the implementation of preventive strategies. ${ }^{8}$ On the other hand, conventional MPI shows an inadequate accuracy in conditions characterized by a diffuse alteration of myocardial perfusion, such as in the case of multivessel CAD causing balanced ischemia, or CAD with superimposed endothelial/microvascular dysfunction due to cardiovascular risk factors.

The recent introduction of dedicated cardiac cameras equipped with Cadmium-Zinc-Telluride (CZT) detectors has revolutionized the field of nuclear cardiac imaging, allowing the consistent reduction of imaging times and patients radiation exposure, while offering increased diagnostic accuracy than conventional Anger cameras in detecting functionally significant CAD. ${ }^{9}$ Due to the increased spatial and energy resolution of these devices, coupled with the linear counting rate response of the detectors, and the capability to acquire data in list mode, CZT cardiac cameras may also allow dynamic 
acquisitions, paving the way for accurate myocardial blood flow (MBF) and flow reserve (MFR) quantification. $^{10,11}$

However, despite initial reports have shown the feasibility of dynamic CZT imaging, with the opportunity of allowing either a better differential diagnosis of patients with suspected CAD or the diagnosis of more precocious stages of the disease, ${ }^{10-12}$ a comprehensive methodological characterization of the technique is still lacking. In particular, the exact duration and timing of the different acquisitions and of the entire imaging protocol is still matter of debate.

In this number of the Journal, Do et al., from the Ottawa Heart Institute, aim at clarifying the appropriate length of time required for dynamic CZT imaging and determining the impact of shortened image acquisition times on image accuracy and repeatability. ${ }^{13}$

Specifically, 29 patients (62\% with known CAD) were submitted to one-day rest-stress MPI on a dedicated cardiac camera equipped with CZT detectors (Discovery NM 530c; GE Healthcare) using ${ }^{99 m}$ Tctetrofosmin as a tracer. Dynamic imaging was performed both on rest and after dipyridamole, and kinetic analysis was accomplished using a dedicated software (FlowQuant software, Vesion 2.5) in order to compute the global left ventricular (LV) K1 uptake rates and K1ratio (K1 stress/K1 rest) using a 1-tissue compartment model. Data were acquired in list mode for 11 minutes both at rest and during stress and then reprocessed in order to simulate reduced acquisition times $(9,7$, and 5 minutes, respectively).

The same analysis was performed with and without motion and attenuation correction in 28 patients, while 27 underwent repeated rest-stress MPI after $18 \pm 13$ days to assess data repeatability.

Reduction of dynamic imaging time resulted in a significant increase of the average $\mathrm{K} 1$ uptake rate both at rest and after stress, but not of the $\mathrm{K} 1$ ratio (K1 stress/K1 rest), independently on whether attenuation or motion correction were performed. Interestingly, while reducing the scan duration to 9 minutes did not result into a significant difference in absolute $\mathrm{K} 1$ values with respect to the standard 11 minutes long acquisition, as image time was reduced further (i.e. to 7 or 5 minutes), the mean K1 difference and standard deviation (SD) increased, likely impairing the accuracy of absolute MBF quantification. The same results were confirmed both for rest and stress acquisitions and, as well as on repeated scans. In brief, reducing dynamic image time to 9 minutes resulted in a mean $4.7 \pm 5.3 \%$ increase in $\mathrm{K} 1$, compared with $11 \pm 8.3 \%$ for a $7 \mathrm{~min}$ scan, and $22 \pm 16 \%$ for a 5 minutes acquisition. Considering that the variation of $\mathrm{K} 1$ values approaches $13 \pm 10 \%$ on repeated scans, it is conceivable that a reduction of the length of dynamic imaging to 7 to 9 minutes would grossly preserve the reliability of MBF estimation. The duration of static acquisition, performed either for patient positioning and residual correction, could be also reduced significantly to 3 minutes prior to rest scan and $30 \mathrm{~s}$ prior to stress scan, with an approximate $1 / 3$ reduction of the total time that a patient is required to be in the scanner (from 32 to 21.5 minutes).

The authors should be commended for their mechanistic study that, despite some shortcuts (i.e. the method for simulating reduced time acquisition), allowed evaluating the reliability of ultrafast dynamic imaging with a commercially available CZT device that may give the possibility to introduce the quantification of MBF and MFR in the daily clinical routine.

This latter aspect is of particular relevance considering the current trend in non-invasive cardiac imaging that, at least in the field of ischemic heart disease, is witnessing a progressive abandonment of stress imaging modalities in favor of CTCA. The main driver of this trend is probably the limited prevalence of obstructive CAD in the current population of patients with suspected ischemic heart disease that come to medical attention, making the elevated negative predictive value of CTCA highly attractive. 5,14

However, the introduction of dedicated cardiac cameras equipped with CZT detectors has increased significantly the diagnostic accuracy of SPECT imaging, particularly in some of the most complex categories of patients, such as those with diffuse CAD. ${ }^{15}$ However, even with those high-end devices, SPECT imaging remains a relative technique, unable to characterize the presence of functional alterations of $\mathrm{MBF}$, which may involve diffusely the LV myocardium as a result of nonobstructive CAD or endothelial/microvascular risk factors. ${ }^{16,17}$

Dynamic imaging is the most obvious solution for this problem, offering obtaining an accurate quantification of MBF and MFR in the setting of a clinically indicated CZT scan. Initial data have demonstrated that, despite the clear technical limitations of ${ }^{99 \mathrm{~m}}$ Tc-labeled radiotracers that show a non-linear myocardial extraction fraction at higher flow rates, the reliability of CZTderived myocardial perfusion parameters is excellent, allowing unmasking the presence of functionally significant CAD at ICA + FFR. ${ }^{10,12}$ Those results might definitively increase the diffusion of CZT devices, particularly in a time-period when the accurate functional characterization of myocardial perfusion is gaining increasing relevance, both in the presence with CAD and in those with suspected coronary microvascular dysfunction. While in the former group of patients the accurate quantification of regional MBF and MFR may be crucial to decide the need of often complex 
interventional procedures, ${ }^{18}$ also in patients with coronary microvascular dysfunction the severity of absolute myocardial perfusion abnormalities is probably the most relevant prognosticator of adverse events. ${ }^{19}$

Finally, this technique would be well suited in the setting of hybrid imaging with combined CTCA and CZT datasets that would allow the accurate depiction of the regional interaction between CAD and downstream myocardial perfusion. ${ }^{13}$

In this regard, the results of Do et al demonstrate that the concept of "fast imaging" can be applied also to dynamic CZT acquisitions, further increasing patient's comfort. Considering that a significant reduction of the waiting intervals between acquisitions can be similarly accomplished without losing diagnostic accuracy, ${ }^{20}$ it might be envisioned that in the near future ultrafast CZT imaging also including dynamic acquisitions will replace conventional MPI, allowing increased diagnostic accuracy and improving patient's comfort.

\section{References}

1. Hachamovitch R, Berman DS, Shaw LJ, Kiat H, Cohen I, Cabico $\mathrm{JA}$, et al. Incremental prognostic value of myocardial perfusion single photon emission computed tomography for the prediction of cardiac death: Differential stratification for risk of cardiac death and myocardial infarction. Circulation. 1998;97:535-43.

2. Members Task Force, Montalescot G, Sechtem U, Achenbach S, Andreotti F, Arden C, Budaj A, et al. ESC guidelines on the management of stable coronary artery disease: The Task Force on the management of stable coronary artery disease of the European Society of Cardiology. Eur Heart J. 2013;2013:2949-3003.

3. Underwood SR, Anagnostopoulos C, Cerqueira M, Ell PJ, Flint EJ, Harbinson M, et al. Myocardial perfusion scintigraphy: The evidence. Eur J Nucl Med Mol Imaging. 2004;31:261-91.

4. Verberne HJ, Acampa W, Anagnostopoulos C, Ballinger J, Bengel $\mathrm{F}$, De Bondt $\mathrm{P}$, et al. EANM procedural guidelines for radionuclide myocardial perfusion imaging with SPECT and SPECT/CT: 2015 revision. Eur J Nucl Med Mol Imaging. 2015;42:1929-40.

5. Neglia D, Rovai D, Caselli C, Pietila M, Teresinska A, AguadéBruix S, et al. Detection of significant coronary artery disease by noninvasive anatomical and functional imaging. Circ Cardiovasc Imaging. 2015;8:e002179. https://doi.org/10.1161/circimaging.11 4.002179 .

6. Danad I, Raijmakers PG, Driessen RS, Leipsic J, Raju R, Naoum $\mathrm{C}$, et al. Comparison of coronary CT angiography, SPECT, PET, and hybrid imaging for diagnosis of ischemic heart disease determined by fractional flow reserve. JAMA Cardiol. 2017;2:1100-7.

7. Chest pain of recent onset: assessment and diagnosis. Clinical guideline [CG95] Published date: March 2010 last updated: November 2016. https://www.nice.org.uk/guidance/cg95.

8. Herscovici R, Sedlak T, Wei J, Pepine CJ, Handberg E, Bairey Merz CN. Ischemia and no obstructive coronary artery disease (INOCA): What is the risk? J Am Heart Assoc. 2018;7:e08868.
9. Gimelli A, Bottai M, Giorgetti A, Genovesi D, Kusch A, Ripoli A, et al. Comparison between ultrafast and standard single-photon emission CT in patients with coronary artery disease: a pilot study. Circ Cardiovasc Imaging. 2011;4:51-8.

10. Zavadovsky KV, Mochula AV, Boshchenko AA, Vrublevsky AV, Baev AE, Krylov AL, et al. Absolute myocardial blood flows derived by dynamic CZT scan vs invasive fractional flow reserve: Correlation and accuracy. J Nucl Cardiol. 2019. https://doi.org/10. 1007/s12350-019-01678-z.

11. Acampa W, Assante R, Mannarino T, Zampella E, D'Antonio A, Buongiorno $\mathrm{P}$, et al. Low-dose dynamic myocardial perfusion imaging by CZT-SPECT in the identification of obstructive coronary artery disease. Eur J Nucl Med Mol Imaging. 2019. h ttps://doi.org/10.1007/s00259-019-04644-6.

12. Agostini D, Roule V, Nganoa C, Roth N, Baavour R, Parienti JJ, et al. First validation of myocardial flow reserve assessed by dynamic 99mTc-sestamibi CZT-SPECT camera: head to head comparison with 15O-water PET and fractional flow reserve in patients with suspected coronary artery disease. The WATERDAY study. Eur J Nucl Med Mol Imaging. 2018;45:1079-90.

13. Do J, Ruddy TD, Wells RG. Reduced acquisition times for measurement of myocardial blood flow with ${ }^{99 \mathrm{~m}}$ Tctetrofosmin and solid-state detector SPECT. J Nucl Cardiol. 2019. https://doi.org/ 10.1007/s12350-019-01678-z.

14. Liga R, Vontobel J, Rovai D, Marinelli M, Caselli C, Pietila M, et al. Multicentre multi-device hybrid imaging study of coronary artery disease: results from the Evaluation of Integrated Cardiac Imaging for the Detection and Characterization of Ischaemic Heart Disease (EVINCI) hybrid imaging population. Eur Heart J Cardiovasc Imaging. 2016;17:951-60.

15. Gimelli A, Liga R, Duce V, Kusch A, Clemente A, Marzullo P. Accuracy of myocardial perfusion imaging in detecting multivessel coronary artery disease: A cardiac CZT study. J Nucl Cardiol. 2017;24:687-95.

16. Gimelli A, Liga R, Pasanisi EM, Casagranda M, Marzullo P. Myocardial ischemia in the absence of obstructive coronary lesion: The role of post-stress diastolic dysfunction in detecting early coronary atherosclerosis. J Nucl Cardiol. 2017;24:1542-50.

17. Liga R, Marini C, Coceani M, Filidei E, Schlueter M, Bianchi M, et al. Structural abnormalities of the coronary arterial wall-in addition to luminal narrowing-affect myocardial blood flow reserve. J Nucl Med. 2011;52:1704-12.

18. Neglia D, Liga R, Caselli C, Carpeggiani C, Lorenzoni V, Sicari $\mathrm{R}$, et al. Anatomical and functional coronary imaging to predict long-term outcome in patients with suspected coronary artery disease: The EVINCI-outcome study. Eur Heart J Cardiovasc Imaging. 2019. https://doi.org/10.1093/ehjci/jez248.

19. Taqueti VR, Shaw LJ, Cook NR, Murthy VL, Shah NR, Foster $\mathrm{CR}$, et al. Excess cardiovascular risk in women relative to men referred for coronary angiography is associated with severely impaired coronary flow reserve. Not obstructive disease. Circulation. 2017;135:566-77.

20. Gimelli A, Liga R, Giorgetti A, Kusch A, Pasanisi EM, Marzullo P. Relationships between myocardial perfusion abnormalities and poststress left ventricular functional impairment on cadmium-zinctelluride imaging. Eur J Nucl Med Mol Imaging. 2015;42:9941003.

Publisher's Note Springer Nature remains neutral with regard to jurisdictional claims in published maps and institutional affiliations. 